\title{
Shigellosis and Changes of Antimicrobial Susceptibility During Six
}

\section{Years}

\author{
Parsa Ghavam, ${ }^{1}$ Maryam Monajemzadeh, ${ }^{1,2}$ Mohammad Taghi Haghi Ashtiani, ${ }^{1,2}$ Setareh Mamishi, ${ }^{1,3}$ \\ Narges Nodeh Farahani, ${ }^{1}$ and Parin Tanzifi, ${ }^{1,2}{ }^{*}$ \\ ${ }^{1}$ Children's Medical Center, Pediatrics Center of Excellence, Tehran, IR Iran \\ ${ }^{2}$ Department of Pathology, Tehran University of Medical Sciences, Tehran, IR Iran \\ ${ }^{3}$ Department of Pediatrics,Tehran University of Medical Sciences, Tehran, IR Iran \\ "Corresponding author: Parin Tanzifi, MD, Department of Pathology, Children's Medical Center Hospital, No 62, Dr. Gharib Street, Keshavarz Boulevard, Tehran, IR Iran. Tel: \\ +98-2161472406; +98-9128152670, Fax: +98-2166948780, E-mail: p-tanzifi@sina.tums.ac.ir
}

Received 2016 January 30; Revised 2016 November 01; Accepted 2017 March 03.

\begin{abstract}
Background: Shigellosis is a diarrheal disease caused by Shigella spp. The majority of cases and deaths occur among children less than 5 years old. In severe cases, antibiotic therapy is recommended to lessen the risk of serious complications and death. Prevalence of different Shigella species and their antibiotic resistance patterns are changing over the time.

Objectives: The current study aimed at assessing the changes in the prevalence of Shigella species and their antibiotic susceptibility among 0- to 14-year-old children referred to Children's Medical Center, Tehran, Iran, from 2009 to 2014.

Methods: The results of stool cultures were retrospectively analyzed to determine the prevalence of different Shigella species, their antibiotic susceptibility patterns and their changes in the largest university affiliated pediatrics center in Tehran.

Results: Among 40700 stool cultures, 507 cases were positive for Shigella spp. Most cases occurred among children under 5 years old. Shigellosis was more prevalent among males. The most common species were Shigella sonnei and Shigella flexneri. In general, they were most sensitive to cefotaxime and most resistant to cotrimoxazole. Resistance to cefotaxime and nalidixic acid increased annually. Resistance to ampicillin had a descending trend. Resistance to cotrimoxazole remained almost constant.

Conclusions: The most common species of Shigella changed from Shigella flexneri to Shigella sonnei over the years. In comparison with the previous studies, resistance to nalidixic acid increased and resistance to ampicillin decreased. The prevalence and antibiotic resistance patterns of Shigella species, at different times and regions are changing. To make appropriate decisions on treatment, it is necessary to monitor the changes.
\end{abstract}

Keywords: Dysentery, Antimicrobial Susceptibility, Pediatrics, Shigella spp

\section{Background}

Shigellosis is a diarrheal disease caused by a genus of Entrobacteriaceae Gram-negative bacteria called Shigella. These bacteria are very invasive, the main route of transmission is fecal-oral. Children younger than 5 years are at a greater risk of infection with Shigella. About $70 \%$ of all Shigellosis episodes and $60 \%$ of deaths caused by these bacteria occur in this age group (1). Shigella genus has 4 species: Shigella dysenteriae (serogroup A), Shigella flexneri (serogroup B), Shigella boydii (serogroup C), and Shigella sonnei (serogroup D) (1-4). Shigella sonnei is more prevalent in the developed countries, while Shigella flexneri is more prevalent in the developing countries (1-3, 5-8). Definite diagnosis of this disease is made by paraclinical tests such as stool exam, stool culture, and serological tests (7, 9). Usually, shigellosis is a self-limited disease, but sometimes severe cases with adverse events are observed $(1,9)$. The mainstay of therapy is rehydration, however in some cases of severe shigellosis, antibiotic therapy is required to shorten the symptomatic period of the disease (5, 6, 8-12). Several studies showed that resistance of Shigella species to the 1st line drugs such as ampicillin and cotrimoxazole is reported worldwide and resistance to other antibiotics is increasing over the time $(7,8,10,13,14)$.

\section{Objectives}

The current study aimed at assessing the changes in the prevalence of Shigella species and their antibiotic susceptibility at children's medical center (CMC) in Tehran, Iran. The purpose was to identify antimicrobial resistance pattern of Shigella spp. to suggest solution for empirical antimicrobial therapy. 


\section{Methods}

The current retrospective study collected the results of stool exam of children 0 to 14 years old submitted from July23, 2009 to August 22, 2014, to the laboratory of CMC hospital, which is one of the tertiary educational pediatric hospitals affiliated to Tehran University of Medical Sciences.

Only 1 Shigella specimen per patient per diarrheal episode was analyzed. The collected data included all stool cultures in the mentioned period. For positive cultures, a form was completed including: demographic data (age, gender, results of laboratory tests, outpatient or inpatient), and paraclinical data consisting of Shigella species, and its antibiotic sensitivity.

Stool specimens were cultured on eosin methylene blue (EMB) and xylose lysine deoxycholate (XLD) agar mediums. A fecal embedded swab was put on SF (Streptococcus faecalis) broth and incubated at $37^{\circ} \mathrm{C}$ for 8 to 12 hours, and then, a specimen was subcultured on XLD agar again. Testing was continued on lactose negative colonies and $\mathrm{H} 2 \mathrm{~S}$ negative samples. Biochemical tests such as growth on triple sugar iron agar (TSI), motility, urease, indole, MRVP [methyl red (MR) and Voges-Proskauer (VP)], citrate agar tests, and reaction for different antisera were done by standard methods on grown bacteria to identify Shigella species. Antibiotic susceptibility testing for ampicillin, cotrimoxazole, cefotaxime, and nalidixic acid was performed in all specimens by disk diffusion method according to the guidelines of the Clinical and Laboratory Standards Institute (CLSI).

The significance of differences in proportions of antimicrobial resistance of Shigella species was determined by the Chi-square or the Fisher exact tests (when the expected value in $>20 \%$ of the cells was $<5$ ). P values $<0.05$ were considered statistically significant. Statistical analysis was performed by SPSS software version 22 .

\section{Results}

Totally, 40,700 stool samples were evaluated, 507 (1.24\%) of which were positive for Shigella species; 471 out of 507 (52.37\%) Shigella spp. isolates were identified as Shigella sonnei which was the most common species of Shigella, followed by Shigella flexneri (37.5\%), Shigella boydii (2.6\%), and Shigella dysenteriae (0.6\%). Figure 1 shows the frequency of Shigella species in each year during the study period. Among the studied cases, 54.4\% were male and $45.6 \%$ female. The most frequent species in both genders was Shigella sonnei. Shigellosis mostly occurred (53.6\%) during autumn. The patients were divided into 2 age groups: group 1 , children $\leq 5$ years; and group 2 , children $>5$ years.
Most cases (64.1\%) were classified in the 1st group. In both age groups, Shigella sonnei was the most prevalent species.

All Shigella spp. isolates were mostly sensitive to cefotaxim (66.2\%) and nalidixic acid (60.2\%); whereas mostly resistant to cotrimoxazle (94.4\%) and ampicillin (68.1\%). Shigella sonnei was mostly sensitive to cefotaxime (66.34\%), nalidixic acid (64.9\%), and ampicillin (52.4\%); and mostly resistant to cotrimoxazole (97.3\%). Shigella flexneri was mostly sensitive to cefotaxime (61.9\%), nalidixic acid (56.1\%); and mostly resistant to ampicillin (96.75\%) and cotrimoxazole (92.1\%). Comparison of the results of susceptibility by year over the study period (Figure 2 ) indicated that the resistance rate to cefotaxime and nalidixic acid increased from $13.9 \%$ to $52.2 \%$ and from $33.3 \%$ to $65.2 \%$, respectively ( $\mathrm{P}$ value $=0.001)$, while resistance rate to ampicillin decreased from $77.1 \%$ to $56.5 \%$ (P value $=0.001$ ). No significant changes were observed in resistance to cotrimoxazole during the study period. A more detailed study among 2 more prevalent species showed that Shigella sonnei resistance to cefotaxime and nalidixic acid had an ascending trend over the years, while its resistance rate to ampicillin had a decreasing trend from $77.1 \%$ to $56.5 \%$ (P value $=0.00$ ). On the other hand, Shigella flexneri resistance increased significantly only to cefotaxime $(P$ value $=0.017)$.

\section{Discussion}

Diarrheal diseases are 1 of the most important and most prevalent diseases among children $(12,15,16)$. Shigella spp. as Gram-negative bacillus is an important etiology of infectious gastroenteritis in humen $(5,6,8,11,12$, 1517). The prevalence of Shigella serogroups is different from one society to another $(2,5,6,8)$. Level of development, general hygiene and socioeconomic conditions, all affect the prevalence of Shigella serogroups $(3,8)$. Studies show that in the developed countries Shigella sonnei is more frequent, while in the developing countries Shigella flexneri is more prevalent $(2,3,5,8)$. The reason is unknown, but there is a hypothesis that links this difference in the prevalence of Shigella serotypes with poor sanitary conditions to stool contamination of drinking water and the ability of Shigella flexneri to be more easily transmitted by drinking water, compared with Shigella sonnei (17). Over time, and with the development of societies, frequency of Shigella serogroups changes $(3,6,11)$. For example, studies in China (8) and Malaysia (6) showed these changes over time. Previous studies conducted in the studied center showed that Shigella flexneri was more prevalent (5), but this pattern changed and now Shigella sonnei is more frequent. This could be due to the quality improvement of health care and socio-economic status. It was found that shigellosis was more prevalent during rainy seasons (autumn), which 


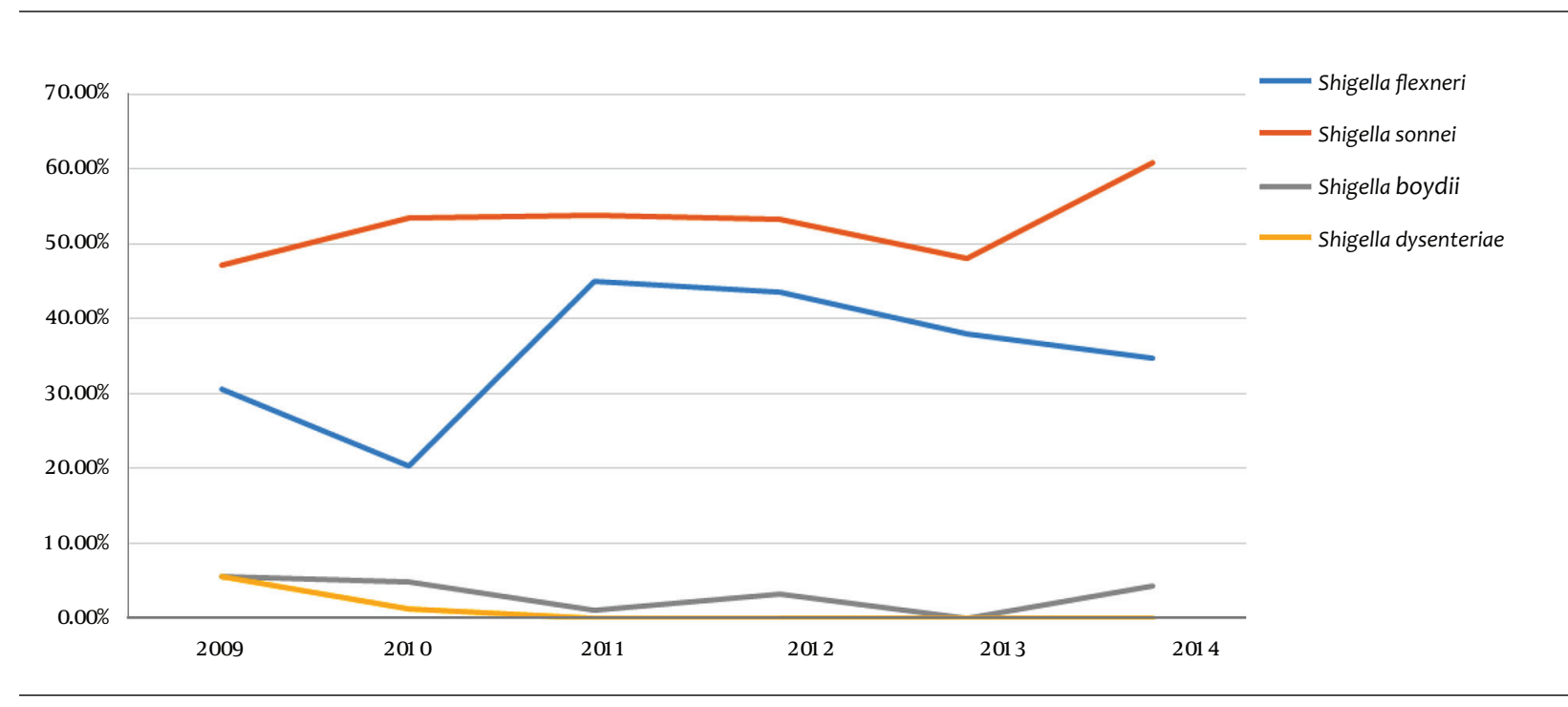

Figure 1. Prevalence of Shigella Serogroups During the Study

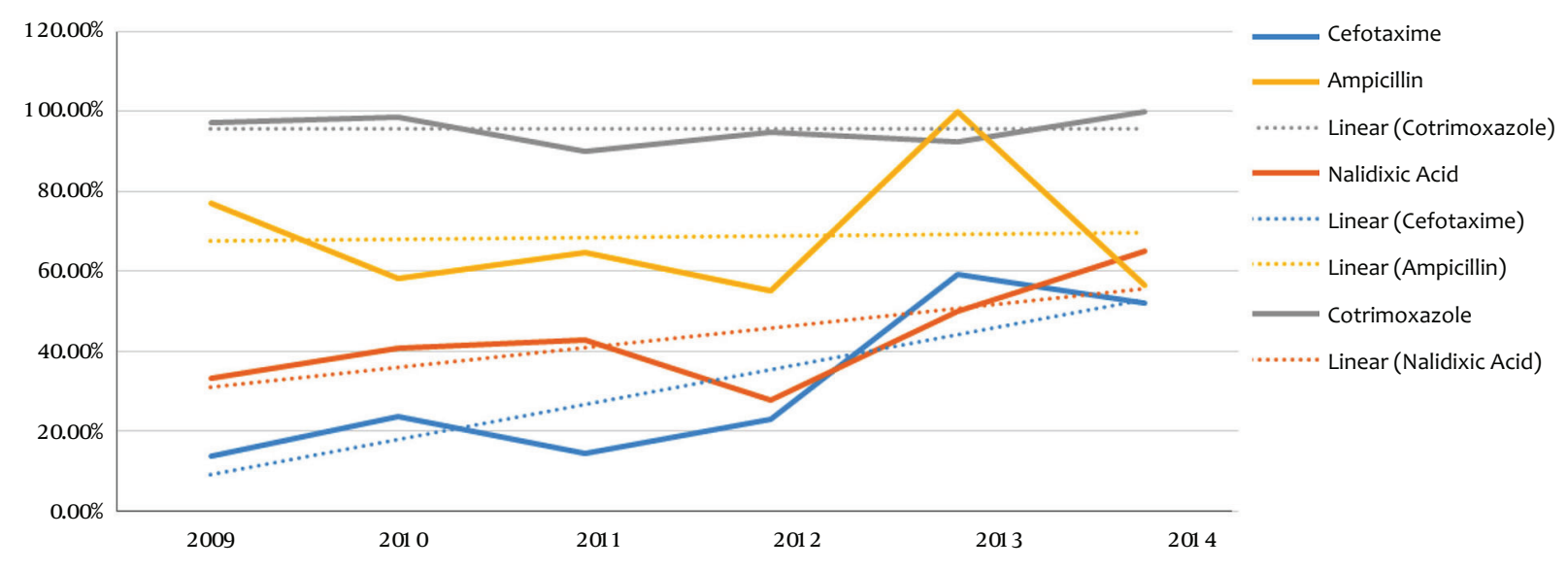

Figure 2. Antibiotic Resistance Trends During the Study

was compatible with the findings of other studies $(6,13,17)$; although in some regions with temperate climate, shigellosis was more prevalent during hot months of the year(11). Infection by Shigella spp. can occur at any age, but children under 5 years old are more prone to being infected by these bacteria $(2,3,6,7,11,12)$. In the current study $64 \%$ of cases were among this age group.

Antibiotic sensitivity pattern of Shigella spp. varies in different countries and is changing over time $(5,6,8$, $11,12)$. It is very important to understand these changes. The current study assessed the sensitivity and resistance of Shigella serogroups to the following 4 antibiotics: cefotaxime, nalidixic acid, ampicillin, and cotrimoxazole. It was found that the samples were generally more sensitive to cefotaxime (66.2\%) and nalidixic acid (60.2\%), and less sensitive to cotrimoxazole (5.6\%) and ampicillin (31.9\%). In the current study, high resistance to cotrimoxazole was reported, which was compatible with the results of other studies (5-7, 10-12, 16), but resistance to ampicillin was different among studies. The resistance rate to ampicillin was different from 9.5\% in Bangladesh (3) to about 90\% in China (8) and Pakistan (7). Comparison of the current study results with that of the previous study in the center (5) indicated that resistance to nalidixic acid increased during these years. In some other studies, increased resistance rate to nalidixic acid was also reported $(7,10,12,17)$, which may be due to overuse of this drug during recent years.

Antibiotic susceptibility was different among Shigella 
serogroups in several studies $(2,5-8,10,11)$. In the current study, Shigella sonnei was more sensitive to cefotaxime and nalidixic acid in comparison with Shigella flexneri. Also, Shigella sonnei was more sensitive to ampicillin than Shigella flexneri. These findings were compatible with the findings of a study conducted in Spain on travelers' diarrhea (10).

Some studies showed that antibiotic resistance is changing as time goes on $(5,17)$. In 2013, centers for disease control and prevention (CDC) published a report outlining the top 18 drug-resistant threats to the United States. These threats were categorized based on the level of concern: urgent, serious, and concerning. In this report Shigella species were categorized as a serious threat (14). The current study found that during the study period, resistance to cefotaxime and nalidixic acid increased from $13.9 \%$ to $52.2 \%$ and from $33.3 \%$ to $65.2 \%$, respectively. Cefotaxime and nalidixic acid were the most effective antibiotics during the past years, but now the resistance to them is increasing. Increased resistance rate to these antibiotics may be due to the extensive use of them in recent years. Another interesting finding was the difference between ampicillin resistance of Shigella sonnei and Shigella flexneri. The current study showed that resistance of Shigella sonnei to ampicillin decreased significantly. On the other hand, the resistance of Shigella flexneri to this drug increased during the same period. In general, among all samples, resistance rate to ampicillin decreased and simultaneously the prevalence of Shigella sonnei increased. In agreement with Pons et al. (10) the current study suggested that the general decrease of ampicillin resistance rate may be due to increased prevalence of Shigella sonnei.

The current study had some limitations. Regarding CLSI guideline, ciprofloxacin for fecal isolates of Shigella spp. should have been tested and reported, but due to supposed adverse effects of this agent in children nalidixic acid was substituted in the routine examinations; however, whenever resistance to other antimicrobial agents was faced, ciprofloxacin was tested as well, but due to the small number of such cases, they were ignored in the statistical analysis.

On the other hand, it is noteworthy that the hospital was a tertiary referral children's center and almost all of the patients with fecal Shigella isolates were hospitalized, regarding this situation, and based on CLSI guidelines oral cephalosporins such as cefixime were not tested in the study. Albeit with patient responses to intravenous cephalosporins, the study could predict, to some extent, their future responses to this agent after being discharged.

\subsection{Conclusions}

Prevalence order of Shigella spp. changed from Shigella flexneri to Shigella sonnei over the years. In comparison with the previous study, resistance to nalidixic acid increased and resistance to ampicillin decreased. The prevalence and antibiotic resistance pattern of Shigella species, at different times and regions, are changing. Monitoring of these changes is necessary for correct treatment decision making. These findings can help to choose efficient drugs for empirical therapy.

\section{Footnote}

Authors' Contribution: Study concept and design: Parin Tanzifi; aquisition of data: Parsa Ghavam and Narges Nodeh Farahani; analysis and interpretation of data: Parsa Ghavam and Parin Tanzifi; drafting of the manuscript: Parsa Ghavam; critical revision: Parin Tanzifi, Setareh Mamishi, and Maryam Monajemzadeh; statistical analysis: Parsa Ghavam; technical and material support: Mohammad Taghi Haghi Ashtiani; supervision: Parin Tanzifi

\section{References}

1. Kliegman RM, Behrman RE, Jenson HB, Stanton BM. Nelson textbook of pediatrics. Elsevier Health Sciences; 2007.

2. Ashkenazi S. Shigella infections in children: new insights. Semin Pediatr Infect Dis. 2004;15(4):246-52. [PubMed: 15494948].

3. Cloeckaert A, Ud-Din AIMS, Wahid SUH, Latif HA, Shahnaij M, Akter M, et al. Changing Trends in the Prevalence of Shigella Species: Emergence of Multi-Drug Resistant Shigella sonnei Biotype $\mathrm{g}$ in Bangladesh. PLOS ONE. 2013;8(12):e82601. doi: 10.1371/journal.pone.0082601.

4. Carroll KC. In: Jawetz, Melnick, and Adelberg's Medical Microbiology. Brooks GF, Carroll KC, Butel JS, Morse SA, Mietzner TA, editors. United States of America: McGraw-Hill; 2013. Enteric Gram-Negative Rods (Enterobacteriaceae)

5. Pourakbari B, Mamishi S, Mashoori N, Mahboobi N, Ashtiani MH, Afsharpaiman S, et al. Frequency and antimicrobial susceptibility of Shigella species isolated in Children Medical Center Hospital, Tehran, Iran, 2001-2006. Braz Jinfect Dis. 2010;14(2):153-7. [PubMed: 20563441]

6. Banga Singh KK, Ojha SC, Deris ZZ, Rahman RA. A 9-year study of shigellosis in Northeast Malaysia: Antimicrobial susceptibility and shifting species dominance. Z Gesundh Wiss. 2011;19(3):231-6. doi: 10.1007/s10389-010-0384-0. [PubMed: 21654922].

7. Zafar A, Hasan R, Nizami SQ, von Seidlein L, Soofi S, Ahsan T, et al. Frequency of isolation of various subtypes and antimicrobial resistance of Shigella from urban slums of Karachi, Pakistan. Int J Infect Dis. 2009;13(6):668-72. doi:10.1016/j.ijid.2008.10.005. [PubMed:19135399]

8. Zhang J, Jin H, Hu J, Yuan Z, Shi W, Yang X, et al. Antimicrobial resistance of Shigella spp. from humans in Shanghai, China, 2004-2011. Diagn Microbiol Infect Dis. 2014;78(3):282-6. doi: 10.1016/j.diagmicrobio.2013.11.023. [PubMed: 24387959].

9. CDC.gov . Shigella - Shigellosis CDC 2016. Available from: http://www. cdc.gov/shigella/index.html.

10. Pons MJ, Gomes C, Martinez-Puchol S, Ruiz L, Mensa L, Vila J, et al. Antimicrobial resistance in Shigella spp. causing traveller's diarrhoea (1995-2010): a retrospective analysis. Travel Med Infect Dis. 2013;11(5):315-9. doi: 10.1016/j.tmaid.2013.06.010. [PubMed: 23886737]. 
11. Ozmert EN, Ince OT, Orun E, Yalcin S, Yurdakok K, Gur D. Clinical characteristics and antibiotic resistance of Shigella gastroenteritis in Ankara, Turkey between 2003 and 2009, and comparison with previous reports. Int J Infect Dis. 2011;15(12):e849-53. doi: 10.1016/j.ijid.2011.08.008. [PubMed: 21982814].

12. Mamatha B, Pusapati BR, Rituparna C. Changing patterns of antimicrobial susceptibility of Shigella serotypes isolated from children with acute diarrhea in Manipal, South India, a 5 year study. Southeast Asian J Trop Med Public Health. 2007;38(5):863-6. [PubMed: 18041303].

13. Dromigny JA, Macondo EA, Juergens-Behr A, Siby T, Perrier-GrosClaude JD. The distribution and antibiotic susceptibility of Shigella isolates in Dakar, Senegal (2000-2002). Int J Antimicrob Agents. 2004;24(3):307-8. doi: 10.1016/j.ijantimicag.2004.04.001. [PubMed 15325440].

14. Centers for Disease Control and prevention . Antibiotic resistance threats in the United States. ; 2013

15. Hien BT, Scheutz F, Cam PD, Serichantalergs O, Huong TT, Thu TM, et al. Diarrheagenic Escherichia coli and Shigella strains isolated from children in a hospital case-control study in Hanoi, Vietnam. J Clin Microbiol. 2008;46(3):996-1004. doi: 10.1128/JCM.01219-07. [PubMed: 18174300].

16. Kosek M, Yori PP, Pan WK, Olortegui MP, Gilman RH, Perez J, et al. Epidemiology of highly endemic multiply antibiotic-resistant shigellosis in children in the Peruvian Amazon. Pediatrics. 2008;122(3):e5419. doi: 10.1542/peds.2008-0458. [PubMed: 18710884].

17. Peleg I, Givon-Lavi N, Leibovitz E, Broides A. Epidemiological trends and patterns of antimicrobial resistance of Shigella spp. isolated from stool cultures in two different populations in Southern Israel. Diagn Microbiol Infect Dis. 2014;78(3):287-91. doi: 10.1016/j.diagmicrobio.2013.12.005. [PubMed: 24418369] 